\title{
O papel da gota citoplasmática na funcionalidade de espermatozoides em mamíferos: uma revisão atualizada
}

The role of cytoplasmic droplet over sperm functionality in mammals: an updated review

\author{
Carlos Mattos Teixeira Soares ${ }^{1}$, Mariana Machado-Neves ${ }^{1}$ \\ ${ }^{1}$ Universidade Federal de Viçosa
}

\section{Resumo}

A gota citoplasmática é uma protuberância de citoplasma geralmente posicionada na peça intermediária dos espermatozoides. É considerada uma organela transitória, uma vez que migra pelo espermatozoide durante o trânsito epididimário, quando é removida dessas células na maioria dos mamíferos. Dúvidas sobre se a presença desta gota está relacionada de forma positiva ou negativa com a função e fertilidade espermática são frequentes, já que sua presença no ejaculado pode afetar a fertilidade espermática e o uso do sêmen em biotécnicas da reprodução. Porém, mesmo não sendo totalmente compreendida a nível molecular e funcional, sabe-se que a gota citoplasmática é necessária ao espermatozoide durante seu processo de maturação no epidídimo. Por isso, o objetivo desta revisão foi abordar pontos sobre a fisiologia e funções das gotas citoplasmáticas, bem como relacionar sua presença com a maturação e fertilidade dos espermatozoides.

Palavras-chave: andrologia animal, maturação espermática, fertilidade, morfologia espermática.

\section{Abstract}

The cytoplasmic droplet is a protuberance of cytoplasm usually positioned in the sperm midpiece. It is considered a transient organelle that migrates through the sperm cell during its epididymal transit when it is finally removed from the cell in most mammals. Doubts over whether the presence of cytoplasmic droplets is positively or negatively related to sperm functions and fertility are common, as its presence in the ejaculate may affect sperm fertility and the use of semen in assisted reproductive technology. Although the understanding of cytoplasmic droplets function is not fully clarified at molecular and functional levels, it is known that cytoplasmic droplet is necessary for spermatozoa, especially during their maturation in the epididymal duct. Therefore, the aim of this review was to discuss aspects of the physiology and function of cytoplasmic droplets, as well as to relate their presence to sperm maturation and their consequent fertility.

Keywords: animal andrology, sperm maturation, fertility, sperm morphology.

\section{Introdução}

A morfologia espermática é um dos parâmetros usados em exames andrológicos para seleção de reprodutores e diagnóstico de casos de sub/infertilidade (Arruda et al., 2015; Love, 2018). Sabe-se que disfunções testiculares e epididimárias podem causar alterações na morfologia da célula espermática, especificamente em acrossoma, cabeça, peça intermediária e cauda (Chen et al., 2019; Díaz et al., 2019). Dentre as patologias espermáticas, cita-se a presença da gota citoplasmática (GC), que é uma massa esférica de citoplasma localizada na peça intermediária que não foi totalmente retirada de espermátides alongadas pelas células de Sertoli ainda no testículo (Hermo et al., 2010; Yuan et al., 2013).

Apesar da GC não ser totalmente compreendida a nível molecular e funcional (Au et al., 2015a), algumas funções já foram relacionadas com esta estrutura celular (Hermo et al., 2010; Cooper, 2011; Xu et al., 2013). Mesmo assim, ainda existem controvérsias e dúvidas sobre se a presença de GC está relacionada de forma positiva ou negativa com a função e fertilidade espermática (Cooper, 2011; Rengan et al., 2012). Por um lado, sua presença no ejaculado pode afetar a fertilidade espermática em animais que apresentam esse remanescente citoplasmático dispensável (Rengan et al., 2012; Yuan et al., 2013; Xu et al., 2013). Por outro lado, a GC representaria uma estrutura funcional e necessária ao espermatozoide, principalmente durante a maturação no trânsito epididimário (Yuan et al., 2013; Xu et al., 2013). Neste

Correspondência: carlosmattos1991@gmail.com

Recebido: 21 de novembro de 2019

Aceito: 23 de junho de 2020 
contexto, esta revisão tem como objetivo discutir alguns pontos sobre a fisiologia e funções das GCs, e relacionar sua presença com a maturação e consequente fertilidade dos espermatozoides.

\section{Biogênese e composição das gotas citoplasmáticas}

Relatada pela primeira vez por Retzius (1909), a GC ou Corpúsculo de Hermes é uma pequena protuberância de citoplasma geralmente posicionada na peça intermediária dos espermatozoides (Rengan et al., 2012; Au et al., 2015a). Ela é considerada uma organela evolutivamente conservada, sendo descrita no sêmen de mais de 30 espécies de vertebrados (Yuan et al., 2013). No entanto, essa organela é transitória e migra pelo espermatozoide durante o trânsito epididimário até ser removida, exceto no homem, espécie que permanecem até após a ejaculação (Xu et al., 2013; Au et al., 2015a).

A formação da GC ocorre no testículo na etapa final da diferenciação das células germinativas durante a espermatogênese, fase denominada de espermiogênese (Oko et al., 1993; Au et al., 2015a). À medida que as espermátides alongadas se movem em direção ao lúmen do túbulo seminífero, os elementos de membrana vesicular e tubular se agrupam formando um aglomerado citoplasmático (Moore et al., 2010). Antes dos espermatozoides serem transportados para o epidídimo, as células de Sertoli realizam um processo conhecido como extrusão citoplasmática e fagocitam a maior parte do citoplasma das células germinativas. O remanescente de citoplasma se torna o que chamamos de GC (Rengan et al., 2012).

Estudos bioquímicos sugerem que as GCs são derivadas do aparelho de Golgi e do retículo endoplasmático (Moore et al., 2010; Xu et al., 2013). Por microscopia eletrônica, é possível detectar na GC morfologia com abundantes discos membranosos achatados e empilhados denominados cisternas, compostos por proteínas estruturais derivadas do aparelho de Golgi (Oko et al., 1993; Moore et al., 2010; $\mathrm{Au}$ et al., 2015a). Proteínas expressas no testículo podem afetar sua formação, como a proteína de maturação espermática 1 que é necessária para a formação normal de GCs (Zheng et al., 2007).

Quanto a sua composição, as GCs são circundadas por uma membrana celular e contêm citoplasma com citosol e citoesqueleto. Elas possuem aproximadamente $2 \mu \mathrm{m}$ de diâmetro e são compostas por lipídios, lipoproteínas, RNAs e uma grande variedade de enzimas hidrolíticas (Cooper, 2011; Rengan et al., 2012). As funções metabólicas e glicolíticas destas, porém, só foram elucidadas recentemente (Yuan et al., 2013; Xu et al., 2013; Au et al., 2015a; Au et al., 2015b; Hermo et al., 2019).

Uma grande variedade de proteínas presentes nas GCs, incluindo enzimas, proteínas do citoesqueleto, proteínas de transporte, glicoproteínas, lipoproteínas, nucleoproteínas, dentre outras, estão envolvidas no metabolismo, motilidade, transporte de membrana e maturação dos espermatozoides (Yuan et al., 2013). Dentre 105 proteínas identificadas nas GCs de camundongos por Yuan et al. (2013), 71 (68\%) são enzimas envolvidas no metabolismo energético. Au et al. (2015a) identificaram 1511 proteínas na GC do rato, das quais a transportadora de glicose 3 (GLUT-3) e hexoquinase 1 (Hex1) foram as mais abundantes (Hermo et al., 2019).

Enzimas como fosfatase alcalina, creatina quinase, superóxido dismutase, Hex1, e as desidrogenases presentes em GCs afetam o metabolismo energético dos espermatozoides (Aitken, 2004; Cooper, 2011; Rengan et al., 2012; Yuan et al., 2013; Au et al., 2015a; Angrimani et al., 2017). Já enzimas conversoras de angiotensina e 15-lipoxigenase participam da maturação espermática, enquanto a roporina tem papel na regulação da motilidade dos espermatozoides (Rengan et al., 2012). Receptores de proteínas como GLUT-3, enzimas glicolíticas, actina filamentosa, TMED e tubulina alfa 1C estão envolvidas no transporte de membrana (Au et al., 2015a). Já os receptores de inositoltrisfosfato e acalreticulina também foram encontrados nas vesículas de GCs, e ambos têm relação com a reação acrossômica (Rengan et al., 2012).

\section{Migração e remoção das gotas citoplasmáticas}

Durante o trânsito epididimário, os espermatozoides sofrem uma alteração morfológica característica, que é a migração da GC. Relatada pela primeira vez por Merton (1939), a migração ocorre do colo ou região proximal da peça intermediária para a região distal da peça intermediária do espermatozoide durante a maturação (Xu et al., 2013; Angrimani et al., 2017). Além disto, as membranas internas giram ao redor do axonema e da bainha mitocondrial à medida que a GC migra (Hermo et al., 2019).

A localização epididimária desta migração varia entre as espécies. Geralmente, esta se inicia na cabeça distal do epidídimo e ocorre até a chegada do espermatozoide no corpo proximal deste órgão 
(Moore et al., 2010; Cooper, 2011; Angrimani et al., 2017). Ainda não é totalmente entendido por que ou por qual mecanismo o movimento da GC ocorre no epidídimo (Moore et al., 2010). Foi sugerido que a migração ocorra a partir de movimentos peristálticos realizados por células mioides da parede externa do ducto epididimário, agindo sobre os espermatozoides em altas concentrações dentro do lúmen (Cooper, 2005; Hermo et al., 2010). Erros durante a migração gerando posicionamentos anormais podem ser um indicativo de falhas durante a espermatogênese (Yuan et al., 2013; Xu et al., 2013).

Simultaneamente à migração, os espermatozoides adquirem motilidade. Assim, a localização normal da GC correlaciona-se com o potencial de motilidade durante a maturação. O mecanismo exato relacionado ao ganho de motilidade ainda é incerto. Porém, sabe-se que a migração proporciona um efeito positivo durante o trânsito do espermatozoide no epidídimo, essencial para que esta célula vença seus desafios no ambiente pós-ejaculatório (Xu et al., 2013; Angrimani et al., 2017).

Em humanos, diferentemente de outras espécies, as GCs parecem não migrar ao longo da peça intermediária, uma vez que tanto os espermatozoides testiculares quanto os do ejaculados têm GCs logo atrás da região da cabeça. Além disto, quando uma GC está aumentada de tamanho, esta é capaz de estender o comprimento da peça intermediária de espermatozoides humanos (Cooper, 2011).

Os espermatozoides perdem suas GCs durante a ejaculação. O mecanismo de eliminação ainda não totalmente esclarecido, mas provavelmente envolve cisalhamento mecânico ou atividades enzimáticas (Cooper, 2005; Moore et al., 2010). Em camundongos, quase todos os espermatozoides $(99,7 \%)$ coletados no útero pós-coito não contêm GC. Considerando que de 75 a $88 \%$ dos espermatozoides da cauda do epidídimo apresentam GC, autores têm sugerido que estas estruturas são removidas durante a ejaculação (Xu et al., 2013). Em algumas espécies como bovinos, equinos e suínos, a remoção também pode estar envolvida com a composição do líquido da vesícula seminal (Cooper, 2005; Heise et al., 2011). Uma hipótese é que a proteína de ligação a fosfolipídios hemolíticos, que foi identificada na ampola e na vesícula seminal de bovinos, mas não no fluido epididimário, possa liberar ou auxiliar a liberação da GC (Thundathil et al., 2001).

Porém, nem todas as GCs são removidas (Hermo et al., 2010), havendo uma grande variação entre as espécies e individual. A falha em eliminar a GC tem sido associada à infertilidade em ratos (Moore et al., 2010). Angulação flagelar é o defeito mais comum quando não ocorre a liberação da gota, principalmente em espécies com flagelos flexíveis, como camundongo e touro (Yeung et al., 2002).

Vários fatores estão envolvidos na remoção da $\mathrm{GC}$, tais como espécie, idade e até mesmo a raça do animal (Carreira et al., 2012; Banaszewska et al., 2015). Foi demonstrado que touros e javalis jovens, que ainda não completaram todo o processo puberal, possuem um percentual elevado de espermatozoides com GCs no ejaculado (Carreira et al., 2012; Banaszewska et al., 2015). No entanto, à medida que atingem a maturidade sexual, a porcentagem de espermatozoides com esta estrutura diminui. Desta forma, quando a GC está presente no espermatozoide de mamíferos domésticos que já passaram pela maturidade sexual, esta é considerada um defeito morfológico, seja quando em posição proximal (defeito maior) ou distal (defeito menor) em relação à cabeça do espermatozoide (Fig. 1) (Barth e Oko 1989; Thundathil et al., 2001; CBRA, 2013).

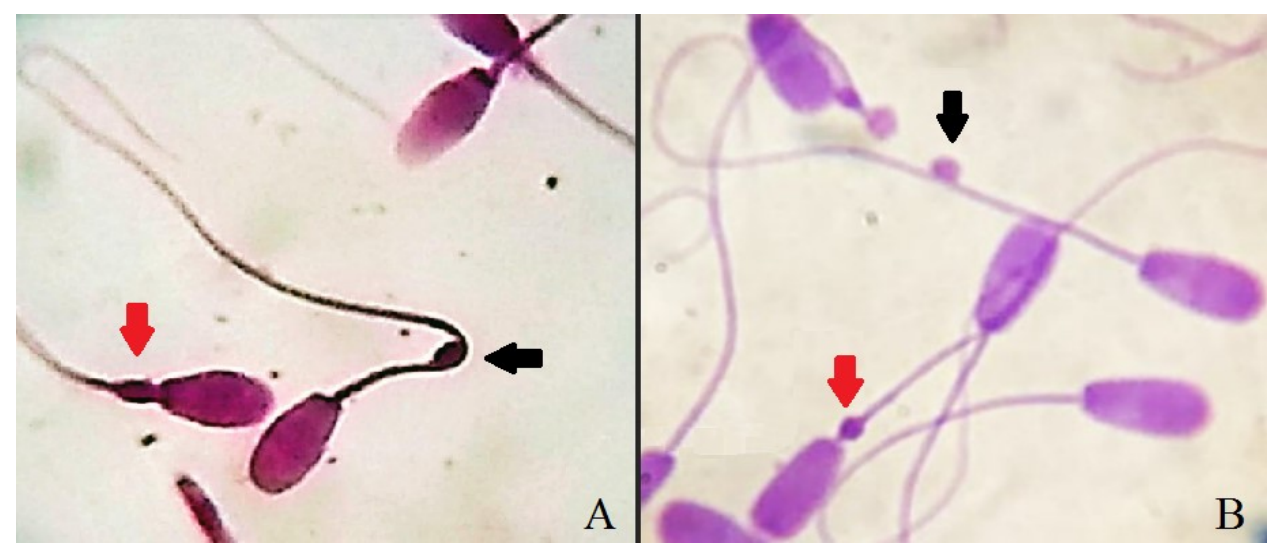

Figura 1. Espermatozoides de ejaculado das espécies equina (A) e bovina (B) apresentando gota citoplasmática em posição distal (seta preta) e proximal (seta vermelha). Coloração: eosina-nigrosina, 1000x. 
Após a remoção, a GC deve possuir um invólucro membranoso intacto, assim como os espermatozoides, que devem reparar sua membrana plasmática no local da remoção (Yuan et al., 2013). Uma membrana intacta é mais provável quando a GC assume uma forma esférica, pois apresenta uma menor área no local de constrição onde ocorre a liberação (Cooper, 2011). Na falha do vedamento da membrana do espermatozoide há a perda de componentes intracelulares necessários para a função espermática, podendo levar à ausência de motilidade (Cooper, 2011).

Diferentemente da maioria das espécies, em humanos a GC está presente no espermatozoide após a ejaculação e não é considerada prejudicial à função espermática (Rengan et al., 2012). As GCs estão presentes em células vivas e móveis no sêmen humano, em meios diluidores, no muco cervical e em espermatozoides recuperados do trato superior feminino (Chantler e Abraham-Peskir, 2004).

\section{Funções realizadas pela gota citoplasmática}

A primeira função relatada para GC foi a de regulação da osmolaridade, que está relacionada à entrada ou saída de água do espermatozoide no epidídimo, mantendo a integridade de membrana (Yuan et al., 2013). Os espermatozoides enfrentam desafios hiposmóticos durante sua jornada (Angrimani et al., 2017), no entanto, eles possuem pouco citoplasma e, por isso, não possuem organelas suficientes para osmorregulação (Rengan et al., 2012). Assim, as GCs contêm canais de osmólitos para facilitar a osmose em conjunto com o epidídimo, sendo responsáveis pela osmoadaptação vital para a função espermática (Carreira et al., 2012; Rengan et al., 2012).

Mais tarde foi observado que a morfologia e localização normais das GCs estão associadas ao metabolismo energético e desenvolvimento de motilidade durante o processo de maturação espermática (Xu et al., 2013; Au et al., 2015a). Porém, o mecanismo exato pelo qual a GC influencia a motilidade espermática não é totalmente elucidado (Xu et al., 2013). A hipótese é que a composição da GC com lipídios, RNAs, lipoproteínas e enzimas hidrolíticas no seu citoplasma estão ligados à atividade mitocondrial do espermatozoide e, consequentemente, a geração de energia necessária (Cooper, 2011; Rengan et al., 2012; Angrimani et al., 2017).

Por fim, as GCs podem também servir como fonte de energia temporária para a produção de energia durante a maturação espermática, suportando eventos moleculares e celulares, regulando o metabolismo energético e a aquisição de motilidade, transição essencial para um espermatozoide imóvel e infértil se tornar móvel e fértil (Yuan et al., 2013; Au et al., 2015a).

\section{Defeitos de gota citoplasmática em espermatozoides}

Defeitos relacionados com GC em espermatozoides podem ocasionar a perda de fertilidade de um macho, uma vez que alteram o padrão da motilidade progressiva nas vias reprodutivas femininas. Os defeitos mais comuns são a retenção da GC, causada por falhas na remoção no momento da ejaculação, e o excesso deste citoplasma residual, que ocorre quando há falhas na remoção durante a espermiogênese (Cooper, 2011; Diaz-Miranda et al., 2019).

A retenção da GC leva a angulação flagelar e até mesmo a defeitos maiores no espermatozoide como a cauda fortemente dobrada. Desta forma, a retenção está associada à infertilidade em grande parte das espécies, como em touros, garanhões, carneiros e cães (Amann et al., 2000; Angrimani et al., 2017). Como os espermatozoides com flagelos defeituosos possuem baixa ou nenhuma motilidade progressiva é pouco provável que atinjam o oócito (Cooper, 2011). As GCs quando persistem no ejaculado podem comprometer a eficiência das biotecnologias reprodutivas e estão associados à infertilidade tanto in vitro quanto in vivo (Amann et al., 2000; Thundathil et al., 2001). Em humanos, mesmo representando uma estrutura normal de espermatozoides ejaculados, as GCs estão associadas à baixa fertilização quando usadas a técnica in vitro (FIV) e a técnica da injeção intracitoplasmática de espermatozoides (ICSI) (Cooper, 2005).

O excesso de citoplasma residual ocorre devido a parada prematura espermiogênese (Cooper et al., 2004) e falhas na extrusão citoplasmática, e está associado a infertilidade, uma vez que pode inibir a motilidade progressiva (Rengan et al., 2012). No homem, o excesso de citoplasma residual contém níveis elevados de enzimas citoplasmáticas que produzem quantidades patológicas de espécies reativas de oxigênio que podem danificar lipídios, proteínas e DNA da membrana do espermatozoide e afetar a motilidade, morfologia e potencial de fertilização do espermatozoide (Cooper, 2011; Rengan et al., 2012). É importante distinguir o excesso de citoplasma residual, quantidade grande de citoplasma em torno da 
peça intermediária do espermatozoide que tem implicações patológicas, das próprias GCs (Cooper, 2011; Rengan et al., 2012). A Organização Mundial da Saúde considera excesso de citoplasma quando maiores que um terço do tamanho da cabeça do espermatozoide (Cooper, 2010).

Além disto, Thundathil et al. (2001) observaram que espermatozoides sem GCs que coexistem no sêmen juntamente com espermatozoides contendo gotas possuem baixa fertilidade. Isto pode ser devido maturação epididimária anormal (Barth e Oko 1989) ou aos efeitos adversos da GC, como a produção de espécies reativas de oxigênio (Thundathil et al., 2001; Díaz-Miranda et al., 2019). Aitken et al. (1996) demonstraram que as espécies reativas de oxigênio são produzidas como consequência do citoplasma retido em espermatozoides e podem ser responsáveis pelo aumento da peroxidação lipídica de espermatozoides próximos e, consequente, perda da integridade estrutural destes (Thundathil et al., 2001; Carreira et al., 2012).

\section{Considerações Finais}

A ausência de GCs em espermatozoides do ejaculado, na maioria das espécies de mamíferos, tem relação positiva com a fertilidade e com o uso de biotecnologias reprodutivas. Porém a GC tem papel importante como organela transitória com funções de osmoadaptação, metabolismo e motilidade, essenciais para maturação do espermatozoide. Assim, espermatozoides ejaculados são aptos à fertilização graças às funções exercidas pelas GCs durante trânsito pelo epidídimo. Defeitos como a retenção da GC ou excesso de citoplasma residual podem alterar a fertilidade, uma vez que influenciam nas funções do espermatozoide. Novos estudos devem ser realizados para o melhor entendimento das interações da GC com o espermatozoide, com o intuito de auxiliar tratamentos para a infertilidade, principalmente relacionada à perda de motilidade espermática.

\section{Agradecimentos}

Aos professores Gilberto Lourenço e Giancarlo Magalhães por cederem as imagens.

\section{Referências}

Aitken RJ, Buckingham DW, Carreras A, Irvine DS. Superoxide dismutase in human sperm suspensions: relationship with cellular composition, oxidative stress, and sperm function. Free Radic Bio Med, v.21, p.495-504, 1996.

Aitken RJ. Founders' Lecture. Human spermatozoa: fruits of creation, seeds of doubt. Reprod Fertil Dev, v.16, p.655-664, 2004.

Amann RP, Seidel GEJr, Mortimer RG. Fertilizing potential in vitro of semen from young beef bulls containing a high or low percentage of sperm with a proximal droplet. Theriogenology, v.54, p.1499$1515,2000$.

Angrimani DSR, Losano DSR, Lucio CF, Veiga GAL, Landim FC, Nichi M, Vannucchi CI. Cytoplasmic droplet acting as a mitochondrial modulator during sperm maturation in dogs. Anim Reprod Sci, v.181, p.50-56, 2017.

Arruda RP, Celeghini ECC, Garcia AR, Santos GC, Leite TG, Oliveira LZ, Lançoni R, Rodrigues MP. Morfologia espermática de touros: interpretação e impacto na fertilidade. Rev Bras Reprod Anim, v.39, n.1, p.47-60, 2015.

Au CE, Hermo L, Byrne E, Smirle J, Fazel A, Simon PH, Kearney RE, Cameron PH, Smith CE, Vali H, Fernandez-Rodriguez J, Ma K, Nilsson T, Bergeron JJ. Expression, sorting, and segregation of Golgi proteins during germ cell differentiation in the testis. Mol Biol Cell, v.26, p.4015-4032, 2015b.

Au CE, Hermo L, Byrne E, Smirle J, Fazel A, Kearney R E, Smith CE, Vali H, FernandezRodriguez J, Simon PH, Mandato C, Nilsson T, Bergeron JJ. Compartmentalization of membrane trafficking, glucose transport, glycolysis, actin, tubulin and the proteasome in the cytoplasmic droplet/Hermes body of epididymal sperm. Open Biol, v.5, p.150080, 2015a.

Banaszewska D, Biesiada-Drzazga B, Andraszek K. Frequency of cytoplasmic droplet depends on the breed and age of insemination boars. Folia Biol (Kraków), v.63, p.9-18, 2015.

Barth AD, Oko RJ. Abnormal morphology of bovine spermatozoa. 1st edition. Iowa State University Press, Ames, Iowa, 1989.

Carreira JT, Mingoti GZ, Rodrigues LH, Silva C, Perri SHV, Koivisto MB. Impact of proximal 
Soares et al. O papel da gota citoplasmática na funcionalidade de espermatozoides em mamíferos: uma revisão atualizada

cytoplazmic droplets on quality traits and in-vitro embryo production efficiency of cryopreserved bull spermatozoa. Acta Vet Scand, v.54, p.1-7, 2012.

CBRA - Colégio Brasileiro de Reprodução Animal. Manual para exame andrológico e avaliação de sêmen animal. 3.ed, 2013.

Chantler E, Abraham-Peskir JV. Significance of midpiece vesicles and functional integrity of the membranes of human spermatozoa after osmotic stress. Andrologia, v.36, p.87-93, 2004.

Chen F, Gao L, Zhou H, Guo L, Chen Q, Gan Y, Sun X, Li Q, Wang K. The association between sperm head elongation and semen quality. Andrology, v.7, p.840-845, 2019.

Cooper TG, Yeung CH, Fetic S, Sobhani A, Nieschlag E. Cytoplasmic droplets are normal structures of human sperm but are not well preserved by routine procedures for assessing sperm morphology. Hum Reprod, v.19, p.2283-2288, 2004.

Cooper TG. Cytoplasmic droplets: The good, the bad or just confusing? Hum Reprod, v.20, p.9-11, 2005.

Cooper TG. Standard procedures. In: WHO laboratory manual for the examination and processing of human semen. 5th edition. Edited by Cooper TG. Cambridge: Cambridge University Press: v.68, p.7-114, 2010.

Cooper TG. The epididymis, cytoplasmic droplets and male fertility. Asian J Androl, v.13, p.130-138, 2011.

Draz-Miranda EA, Maitan PP, Machado TP, Camilo BS, Lima DA, Okano DS, Penitente-Filho JM, Machado-Neves M, Oliveira LL, Guimarães SEF, Costa EP, Guimarães JD. Disruption of bovine sperm functions in the presence of aplastic midpiece defect. Andrology, v.8, p.201-210, 2019.

Heise A, Thompson PN, Gerber D. Influence of seminal plasma on fresh and post-thaw parameters of stallion epididymal spermatozoa. Anim Reprod Sci, v.123, p.192-201, 2011.

Hermo L, Oliveira RL, Smith CE, Au CE, Bergeron JJM. Dark side of the epididymis: tails of sperm maturation. Andrology, v.7, p.566-580, 2019.

Hermo L, Pelletier RM, Cyr DG, Smith CE. Surfing the wave, cycle, life history, and genes/proteins expressed by testicular germ cells. Microsc Res Tech, v.73, p.320-363, 2010.

LOVE CC. Sperm quality assays: How good are they? The horse perspective. Anim Reprod Sci, v.194, p.63-70, 2018.

Merton H. Studies on reproduction in the albino mouse. II. Contributions on the maturation of the sperm cells. Proc R Soc, v.59, p.145-152, 1939.

Moore K, Lovercamp K, Feng D, Antelman J, Sutovsky M, Manandhar G, van Leyen K, Safranski T, Sutovsky P. Altered epididymal sperm maturation and cytoplasmic droplet migration in subfertile male Alox15 mice. Cell Tissue Res, v.340, p.569-581, 2010.

Oko R, Hermo L, Chan PT, Fazel A, Bergeron JJ. The cytoplasmic droplet of rat epididymal spermatozoa contains saccular elements with Golgi characteristics. J Cell Biol, v.123, p.809-821, 1993.

Rengan AK, Agarwal A, van der Linde M, du Plessis SS. An investigation of excess residual cytoplasm in human spermatozoa and its distinction from the cytoplasmic droplet. Reprod Biol Endocrinol, v.10, p.92, 2012.

Retzius G. Die Spermien der Huftiere. Niol Untersuch, v.14, p.163-178, 1909.

Thundathil J, Palasz AT, Barth AD, Mapletoft RJ. The use of in vitro fertilization techniques to investigate the fertilizing ability of bovine sperm with proximal cytoplasmic droplets. Anim Reprod Sci, v.65, p.181-192, 2001.

Xu H, Yuan SQ, Zheng Z H, Yan W. The cytoplasmic droplet may be indicative of sperm motility and normal spermiogenesis. Asian J Androl, v.15, p.799-805, 2013.

Yeung CH, Anapolski M, Cooper TG. Measurement of volume changes in mouse spermatozoa using an electronic sizing analyzer and a flow cytometer: validation and application to an infertile mouse model. J Androl, v.23, p.522-528, 2002.

Yuan S, Zheng H, Zheng Z, Yan W. Proteomic analyses reveal a role of cytoplasmic droplets as an energy source during epididymal sperm maturation. Plos One, v.8, p.77466, 2013.

Zheng H, Stratton CJ, Morozumi K, Jin J, Yanagimachi R, Yan W. Lack of Spem1 causes aberrant cytoplasm removal, sperm deformation, and male infertility. Proc Natl Acad Sci, USA, v.104, p.68526857, 2007. 